\title{
Subdominant Dark Matter sterile neutrino resonant production in the light of PLANCK
}

\author{
L. A. Popa, D. Tonoiu \\ Institute of Space Science, \\ Bucharest-Magurele, Ro-077125 Romania \\ E-mail: lpopa@spacescience.ro, tonoiud@spacescience.ro
}

\begin{abstract}
Few independent detections of a weak X-ray line at an energy of $\sim 3.5 \mathrm{keV}$ seen toward a number of astrophysical sites have been reported. If this signal will be confirmed to be the signature of decaying DM sterile neutrino with a mass of $\sim 7.1 \mathrm{keV}$, then the cosmological observables should be consistent with its properties.

In this paper we make a coupled treatment of the weak decoupling, primordial nucleosynthesis and photon decoupling epochs in the sterile neutrino resonant production scenario, including the extra radiation energy density via $N_{\text {eff }}$. We compute the radiation and matter perturbations including the full resonance sweep solution for $\nu_{\alpha} / \bar{\nu}_{\alpha} \rightarrow \nu_{s}$ flavor conversion in the expanding Universe.

We show that the cosmological measurements are in agreement with subdominant Dark Matter sterile neutrino resonant production with following parameters (errors at 95\% CL): mass $m_{\nu_{s}}=6.08 \pm 3.22 \mathrm{keV}$, mixing angle $\sin ^{2} 2 \theta<5.61 \times 10^{-10}$, lepton number per flavor $L_{4}=1.23 \pm 0.04\left(L_{4} \equiv 10^{4} L_{\nu_{a}}\right)$ and sterile neutrino mass fraction $f_{\nu_{s}}<0.078$.

Our results are in good agreement with the sterile neutrino resonant production parameters inferred in Ref. [62] from the linear large scale structure constraints to produce full Dark Matter density.
\end{abstract}

Keywords: cosmology: cosmic microwave background, cosmological parameters, early universe, neutrino 


\section{Contents}

1 Introduction 1

2 Sterile neutrino resonant production calculations 3

2.1 Lepton number depletion and evolution of resonance energies 4

2.2 Phase-space distributions 5

2.3 Effects of DM sterile neutrino resonant production on $N_{\text {eff }} \quad 6$

$\begin{array}{lll}3 & \text { Cosmological constraints } & 7\end{array}$

$\begin{array}{lll}3.1 & \text { Method and datasets } & 7\end{array}$

3.2 Analysis 10

$\begin{array}{lll}\text { 3.2.1 Neutrino masses and mixing } & 11\end{array}$

$\begin{array}{lll}3.2 .2 & { }^{4} \mathrm{He} \text { abundance yield } & 11\end{array}$

\section{Introduction}

With about $25 \%$ of the total energy density of the Universe represented by Dark Matter (DM) and six basic cosmological parameters, the $\Lambda \mathrm{CDM}$ cosmological standard model is remarkably successful at reproducing the large-scale structure (LSS) of the Universe $[1,2]$. Despite its successes on large-scales, this model produces too much power at small scales. In general, the observed structures are less abundant ("missing satellite problem") [3-6], have cored density profiles ("core/cusp problem") [7-9] and are too dense ("too-big-to-fail problem") $[10,11]$ than those predicted by the $\Lambda$ CDM model. Numerous attempts involving collisionless DM [4, 12-15] fail to resolve these problems, suggesting that DM may exhibit gravitational properties as well.

The nature of DM remains however unknown and the explanation of its existence requires the revision of Standard Model (SM) of particle physics. In this regard, many extensions of SM have been considered [16-18].

Among the highly motivated candidates for DM is the right-handed sterile neutrino [1922]. The minimal extension of SM by the addition of three right-handed sterile neutrinos (the $\nu \mathrm{MSM}$ ), each one corresponding to a SM active neutrino flavor, can simultaneously explain the active neutrino oscillations and the DM properties [23, 24]. Within this theory, the DM sterile neutrino masses are in the $\mathrm{keV}$ range and the mass of the lightest active neutrino is smaller than $10^{-5} \mathrm{eV}$. This excludes the degenerate mass spectra of the active neutrinos and fixes the absolute mass scale of the other two active neutrinos [25]. Moreover, the $\nu$ MSM can also explain the matter-antimatter asymmetry of the Universe [24].

In the original Dodelson-Widrow (DW) model [26], sterile neutrinos are produced in the early Universe through non-resonant oscillations (NRP) with active neutrinos in presence of negligible leptonic asymmetry. As the DW sterile neutrino is a Warm Dark Matter (WDM) particle with non-negligible velocity dispersion, this suppress the matter power spectrum bellow the free-streaming scale, that affects the DM distribution at small-scales.

For NRP case, the universal Tremaine-Gunn bound [27] on sterile neutrino mass leads to 
$m_{s}^{\mathrm{NRP}}>1.8 \mathrm{keV}$. Lower limits on sterile neutrino mass have been placed from various observational probes. The combination of CMB measurements, LSS and Ly- $\alpha$ forest power spectra lead to $m_{s}^{\mathrm{NRP}}>1.7 \mathrm{keV}$ with a further improvement to $m_{s}^{\mathrm{NRP}}>3 \mathrm{keV}$ when highresolution Lyman- $\alpha$ power spectra are considered [28]. By modeling the SDSS Lyman- $\alpha$ forest flux spectrum, a lower limit of $m_{s}^{\mathrm{NRP}} \geq 13 \mathrm{keV}$ have been found $[29,30]$. The same lower limit has been placed by requiring the number of subhalos in N-body simulation to be larger than the number of the observed dwarf spheroidal galaxies (dSphs) of the Milky Way [31]. Using the phase-space densities derived for dwarf satellite galaxies of the Milky Way a recent work [32] found a lower limit of DW sterile neutrino mass of $m_{s}^{\mathrm{NRP}}>2.5 \mathrm{keV}$.

Sterile neutrino with a keV mass is known to decay radiatively into a photon and an active neutrino, producing a narrow X-ray line with an energy $E_{c}=\frac{1}{2} m_{s}$ [19]. The width of this decay line increases as the fifth power of sterile neutrino mass and as square of its mixing angle, being potentially detectable in various astrophysical DM sources like X-ray background, galaxy clusters, nearby galaxies and our own Milky Way [25]. Bounds on sterile neutrino radiative decay have been used to place upper limits on sterile neutrino mass. Strong upper limit constraints in the NRP scenario are obtained from the observations of Andromeda galaxy (M31) by XMM-Newton $\left(m_{s}^{\mathrm{NRP}}<3.5 \mathrm{keV}\right)[33,34]$ and Chandra $\left(m_{s}^{\mathrm{NRP}}<2.2 \mathrm{keV}\right)$ telescopes [35]. When combined, the upper and lower limits appears to rule out the sterile neutrino NRP scenario.

The DM sterile neutrino resonant production (RP) via resonant Mikheyev-SmirnovWolfenstein (MSW) $[36,37]$ conversion of active neutrinos to sterile neutrinos through the Shi-Fuller mechanism [38] in the presence of leptonic asymmetry has also been proposed $[19,20,39]$. Although the SM of particle physics predicts the leptonic asymmetry of the same order as the baryonic asymmetry, $L \approx B \sim 10^{-10}$, there are scenarios in which much larger leptonic asymmetry can be produced [40-42].

The Tremaine-Gunn bound on sterile neutrino mass for the RP case leads to $m_{s}^{\mathrm{RP}}>1 \mathrm{keV}$ [34]. Also, the combined analysis of WMAP5 and Ly- $\alpha$ data indicates a bound of $m_{s}^{\mathrm{RP}}>2$ $\mathrm{keV}$ in the frame of mixed Cold plus Warm Dark Matter (CWDM) model [43].

Sterile neutrino produced via RP mechanism was also forecast to have a radiative decay mode that could be detectable by X-ray telescopes as an emission line in spectra of X-ray clusters and galaxies [19, 44].

Other proposed mechanisms are sterile neutrino production via interactions with the inflaton field [45-47] and by the decay of heavy scalar singlets [48, 49].

Recently, few independent detections of a weak X-ray emission line at an energy of $\sim 3.5$ $\mathrm{keV}$ have been found in a stacked XMM-Newton spectrum of 73 galaxy clusters with redshifts in the range $0.01-0.35$, in Chandra ACIS-I and ACIS-S spectra of Perseus [50] and in the XMM-Newton spectra of Andromeda galaxy and Perseus galaxy cluster [51]. Evidences of this emission have been also searched in Milky Way with data from Chandra [52] and XMMNewton [53, 54], in deep Suzaku X-ray spectra of the central regions of Perseus, Coma, Virgo and Ophiuchus clusters [55,56], in the stacked XMM-Newton spectra of dwarf spheroidal galaxies in the vicinity of Milky Way [57] and in the stacked spectra of a sample of galaxies selected from Chandra and XMM-Newton public archives[58]. Until now, none of these searches were able to establish precisely if the origin of this line is is a signature of decaying dark matter [51] or it is related to any known atomic transition in thermal plasma, with special interest in Potassium and Chlorine atomic transitions [52-54]. The evidence (or lack of evidence in some cases) of this emission line in different astrophysical sites placed strong constraints on both hypothesis, raising several controversies on this subject [59-61]. 
Sterile neutrinos produced through a resonant mechanism remains however the simplest model for $\mathrm{DM}$ origin of the undefined $\sim 3.5 \mathrm{keV}$ of $\mathrm{X}$-ray line. The $\mathrm{RP}$ sterile neutrino parameters required to produce this signal have been recently inferred from the linear large scale structure constraints to produce full DM density [62]. These parameters are consistent with the Local Group and high-z galaxy count constraints, fulfilling previously determined requirements to successfully solve "missing satellite problem" and "too-big-to-fail problem".

The cosmological constraints on subdominant RP sterile neutrino parameters have not yet derived. The goal of this paper is to place constraints on RP sterile neutrino parameters in a $\Lambda$ CWDM model containing a mixture of WDM in the form of RP sterile neutrino and CDM, by using most of the present cosmological measurements.

In particular, the reconstructed gravitational lensing potential power spectrum obtained by the PLANCK satellite has impact on cosmological parameter degeneracies when DM sterile neutrino scenario is considered [63], offering several advantages against other methods [2, 64]. As the gravitational lensing effect depends on the DM distribution in the Universe, no assumption on light-to-mass bias is required. The projected gravitational lensing potential is sensitive to the matter distribution out to high redshifts, preventing from non-linear corrections required only at very small scales. In addition, unlike the galaxy clustering and the Ly- $\alpha$ forest, the projected gravitational lensing potential probes a larger range of angular scales, most of the signature coming from large scales. Moreover, the preference of present cosmological data for a small but significant leptonic asymmetry have been recently found [65].

This paper is organized as follows. In Sec. 2 we briefly review the DM sterile neutrino RP calculations. In Sec. 3 we describe the model, methods used in our analysis and the datasets we consider. We present our results in Sec. 4 where we examine the consistency and cosmological implications of DM sterile neutrino RP scenario. In Sec. 5 we draw our conclusions.

\section{Sterile neutrino resonant production calculations}

The DM sterile neutrinos with rest mass $m_{s} \geq 1 \mathrm{keV}$ are produced through neutrino oscillations at temperatures close to the QCD phase transition $[19,20]$. The oscillation process take place because the neutrino mass eigenstate components propagate differently as they have different energies, momenta and masses. Eigenstates of neutrino interaction include the active neutrinos/antineutrinos, $\nu_{\alpha} / \bar{\nu}_{\alpha}(\alpha=e, \mu, \tau)$, which are created and destroyed in the $\mathrm{SM}$ of particle physics by weak interactions as well as the sterile neutrinos/antineutrinos, $\nu_{s} / \bar{\nu}_{s}$, which do not participate in weak interactions.

For the purpose of this work we consider three active neutrino flavors $\nu_{\alpha} / \bar{\nu}_{\alpha}$ and one sterile neutrino $\nu_{s} / \bar{\nu}_{s}$ with the production channel $\nu_{\alpha} / \bar{\nu}_{\alpha} \rightarrow \nu_{s} / \bar{\nu}_{s}$. In the early Universe, this conversion can occur through the medium-enhanced MSW resonant mechanism in presence of an initial neutrino lepton number residing in the active neutrinos [19, 20,38]:

$$
L_{\nu_{\alpha}} \equiv \frac{\left(n_{\nu_{\alpha}}-n_{\left.\bar{\nu}_{\alpha}\right)}\right.}{n_{\gamma}}
$$

where $n_{\nu_{\alpha}} / n_{\bar{\nu}_{\alpha}}$ and $n_{\gamma}$ are the active neutrino/antineutrino and photon number densities respectively, $n_{\gamma}=2 \zeta(3) T_{\gamma}^{3} / \pi^{2}$ and $\zeta(3)$ is Riemann zeta function of 3 .

Above the neutrino weak decoupling $\left(T_{d e c} \sim 1 \mathrm{MeV}\right)$, the weak interaction rates of neutrino 
scattering are much faster than the Hubble expansion rate and the neutrino energy distribution can reshuffle into a thermal Fermi-Dirac spectrum. After $T_{\text {dec }}$, when active neutrinos decouple from the thermal plasma, the conversion process can be resonantly enhanced [66], affecting the primordial nucleosynthesis $(T \sim 0.1 \mathrm{MeV})$ and the photon decoupling $(T \sim 0.2$ $\mathrm{eV})$ epochs $[67,68]$.

In this section we follow the detailed resonant production calculations first performed in Refs. $[19,66]$ for adiabatic and continuously sweeping active-sterile resonance, extended then to the non-adiabatically sweeping case in Refs. $[69,70]$ for the computation of the primordial nucleosynthesis abundance yields (the full solution) and examine how the sterile neutrino resonant production affects the photon decoupling.

\subsection{Lepton number depletion and evolution of resonance energies}

The MSW condition for the resonant scaled neutrino momentum $\epsilon_{\text {res }}=p /\left.T\right|_{\text {res }}$ is given by:

$$
\epsilon_{\text {res }} \approx\left(\frac{\delta m^{2} \cos 2 \theta}{4 \sqrt{2} \zeta(3) \pi^{-2} G_{F} \mathcal{L}_{\nu_{\alpha}}}\right) T^{-4},
$$

where $\delta m^{2}=m_{2}^{2}-m_{1}^{2} \approx m_{2}^{2}$ is the difference of the squares of sterile neutrino and active neutrino eigenvalues, $T$ is the photon/plasma temperature, $G_{F}$ is the Fermi constant, $\theta$ is the vacuum mixing angle and $\mathcal{L}_{\nu_{\alpha}}$ is the potential lepton number corresponding to an active neutrino flavor $\alpha$ :

$$
\mathcal{L}_{\nu_{\alpha}} \equiv 2 L_{\nu_{\alpha}}+\sum_{\beta \neq \alpha} L_{\nu_{\beta}}, \quad \beta=(e, \mu, \tau) .
$$

The leptonic asymmetry is most conveniently measured by the neutrino degeneracy parameter [71-73] defined as $\xi_{\nu_{\alpha}}=\mu_{\nu_{\alpha}} / T_{\nu}$, where $\mu_{\nu_{\alpha}}$ is the neutrino chemical potential and $T_{\nu}$ is the present temperature of the neutrino background $\left[T_{\nu} / T_{\gamma}=(4 / 11)^{1 / 3}\right]$. The relation between the lepton number of neutrino flavor $\alpha$ and the corresponding chemical potential is given by:

$$
L_{\nu_{\alpha}}=\left(\frac{1}{12 \zeta(3)}\right)\left(\frac{T_{\nu}}{T_{\gamma}}\right)^{3}\left[\pi^{2} \xi_{\nu_{\alpha}}+\xi_{\nu_{\alpha}}^{3}\right] .
$$

The evolution of the neutrino flavor asymmetries with the temperature is driven by the active neutrino oscillation mixing parameters $[74,75]$. These parameters are accurately measured with the exception of one mixing angle, $\theta_{13}$, that only recently started to be constrained. Actually, the value of $\theta_{13}$ plays a decisive role in determining the temperature at which the equilibrium among active neutrino flavor asymmetries is reached [76,77]. For values of $\theta_{13}$ closed to the present experimental upper bound, it is shown that the active neutrino oscillations are effective at a temperature $T \sim 8 \mathrm{MeV}[78]$ and therefore the active neutrinos reach the chemical equilibrium before BBN. For this reason we consider in the present work the same degeneracy parameter for all neutrino flavors: $\xi_{\nu_{e}}=\xi_{\nu_{\mu}}=\xi_{\nu_{\tau}}$.

As the universe expands, the temperature falls causing the resonance to sweep from low to higher values of the resonant scaled neutrino momentum $\epsilon_{\text {res }}$. This process converts $\nu_{\alpha} / \bar{\nu}_{\alpha}$ to $\nu_{s}$, reducing the potential lepton number $\mathcal{L}_{\nu_{\alpha}}$. As discussed in Refs.[19, 38], the evolution of $\mathcal{L}_{\nu_{\alpha}}$ is dictated by the resonance sweep rate and the dimensionless adiabaticity parameter $\gamma$ given by:

$$
\gamma \approx \frac{\sqrt{5} \zeta(3)^{3 / 4}}{2^{1 / 8} \pi^{3}} \frac{\left(\delta m^{2}\right)^{1 / 4} m_{p l} G_{F}^{3 / 4}}{g^{1 / 2}} \frac{\sin ^{2} 2 \theta}{\cos ^{7 / 4} 2 \theta} \mathcal{L}_{\nu_{\alpha}}{ }^{3 / 4} \epsilon^{-1 / 4} \times\left|1+\frac{\dot{g} / g}{3 H}-\frac{\dot{\mathcal{L}_{\nu_{\alpha}}} / \mathcal{L}_{\nu_{\alpha}}}{3 H}\right|^{-1},
$$


where $m_{p l}$ is the Planck mass, $H$ is the Hubble expansion rate and $g$ is the total statistical weight for relativistic species in the early Universe. Large values of $\gamma(\gamma>1)$ result when the oscillation length is smaller than the resonance width, leading to efficient flavor transformation. If the time rate change of $\mathcal{L}_{\nu_{\alpha}}$ is larger than the Hubble expansion rate, the evolution is non-adiabatic with $\gamma<1$. The likelihood probability of a neutrino at resonance to make the jump between the mass eigenstates is given by Landau-Zerner probability, $P_{L Z}=\exp (-\pi \gamma / 2)$ [79]. The evolution of the potential lepton number when the resonant active neutrino/antineutrino momentum sweeps from 0 to $\epsilon_{\text {res }}$ is then given by:

$$
\mathcal{L}_{\nu_{\alpha}}\left(\epsilon_{\text {res }}\right)=\mathcal{L}^{\text {init }}-\frac{1}{2 \zeta(3)}\left(\frac{T_{\nu}}{T_{\gamma}}\right)^{3} \int_{0}^{\epsilon_{r e s}}\left(1-e^{-\pi \gamma / 2}\right)\left(f_{\nu_{\alpha}}+f_{\bar{\nu}_{\alpha}}\right) \mathrm{d} x,
$$

where $f_{\nu_{\alpha}}$ and $f_{\bar{\nu}_{\alpha}}$ are the initial neutrino/antineutrino Fermi-Dirac distribution functions:

$$
f_{\nu_{\alpha}}(\epsilon)=\frac{1}{F_{2}\left(\xi_{\nu_{\alpha}}\right)} \frac{\epsilon^{2}}{e^{\epsilon-\xi_{\nu_{\alpha}}}+1}, \quad f_{\bar{\nu}_{\alpha}}(\epsilon) \frac{1}{F_{2}\left(-\xi_{\nu_{\alpha}}\right)} \frac{\epsilon^{2}}{e^{\epsilon+\xi_{\nu_{\alpha}}}+1},
$$

and $F_{2}\left( \pm \xi_{\nu_{\alpha}}\right)$ are the relativistic Fermi integrals of second order:

$$
F_{2}\left( \pm \xi_{\nu_{\alpha}}\right) \equiv \int_{0}^{\infty} \frac{x^{2}}{e^{x \mp \xi_{\nu_{\alpha}}+1}} \mathrm{~d} x
$$

\subsection{Phase-space distributions}

The time evolution of the sterile neutrino phase-space distribution, $f_{\nu_{s}}$, can be described by the classical Boltzmann equation [19, 20]:

$$
\frac{\partial}{\partial t} f_{\nu_{s}}(\epsilon)-H(t) \epsilon \frac{\partial}{\partial \epsilon} f_{\nu_{s}}(\epsilon) \approx \Gamma\left(\nu_{\alpha} \rightarrow \nu_{s}\right)\left[f_{\nu_{\alpha}}(\epsilon)-f_{\nu_{s}}(\epsilon)\right],
$$

where: $f_{\nu_{\alpha}}$ is the active neutrino phase-space distribution, $H(t)$ is the Hubble expansion rate and $\Gamma\left(\nu_{\alpha} \rightarrow \nu_{s}\right)$ is the sterile neutrino effective production rate:

$$
\Gamma\left(\nu_{\alpha} \rightarrow \nu_{s}\right) \approx \frac{1}{2} \Gamma_{\nu_{\alpha}}(p, T)\left\langle P_{m}\left(\nu_{\alpha} \rightarrow \nu_{s}\right)\right\rangle,
$$

where $\Gamma_{\nu_{\alpha}}$ is the collision rate:

$$
\Gamma_{\nu_{\alpha}}(p, T) \approx\left\{\begin{array}{l}
1.27 G_{F}^{2} p T^{4}, \alpha=e \\
0.92 G_{F}^{2} p T^{4}, \alpha=\mu, \tau
\end{array}\right.
$$

The average oscillation probability $\left\langle P_{m}\left(\nu_{\alpha} \rightarrow \nu_{s}\right)\right\rangle$ is given by:

$$
\left\langle P_{m}\left(\nu_{\alpha} \rightarrow \nu_{s}\right)\right\rangle=\frac{1}{2} \frac{\Delta^{2}(p) \sin ^{2} 2 \theta}{\Delta^{2}(p) \sin ^{2} 2 \theta+D^{2}(p)+\left[\Delta(p) \cos 2 \theta+V^{L}-V^{T}\right]^{2}}
$$

where $\Delta(p)=\delta m^{2} / 2 p$ is the vacuum oscillation factor, $D(p)=\Gamma_{\nu_{\alpha}}(p) / 2$ is the quantum damping rate, $V^{T}$ is the thermal potential and $V^{L}$ is the asymmetric lepton potential:

$$
V^{L}=2 \sqrt{2} \zeta(3) \pi^{-2} G_{F} T^{3} \mathcal{L}_{\alpha} .
$$

For temperatures characteristic to the post weak decoupling era $(\mathrm{T}<3 \mathrm{MeV})$ the contribution of the thermal potential $V^{T}$ in Eq. (2.11) is very small and can be neglected. 
Starting from $\nu_{\alpha} / \bar{\nu}_{\alpha}$ thermal distributions at a scale factor $a / a_{0} \sim 10^{-13}$ ( $a_{0} \equiv 1$ today), corresponding to a temperature $T \simeq 133 \mathrm{MeV}\left(m_{s} / 1 \mathrm{keV}\right)^{1 / 3}$ (near sterile neutrino maximum rate production temperature), we simultaneously evolve Eqs. (2.2), (2.5), (2.6) and (2.9) to obtain the evolution of $\mathcal{L}_{\nu_{\alpha}}\left(\epsilon_{\text {res }}\right)$ in the expanding Universe for the entire range of resonant scaled neutrino momentum values. In this calculation we neglect the effects of the QCD quark-gluon phase transition that may disturb the DM sterile neutrino production rate by the alteration of the time-temperature relationship in the early Universe (due to variation of the statistical weight in different particles) [19,63].

Figure 1 presents the dependence of $\epsilon_{r e s} \mathcal{L}_{\nu_{e}}\left(\epsilon_{r e s}\right)$ on sterile neutrino masses and mixing angles for two different initial lepton numbers, $L_{4}=12$ and 24 (we define $L_{4} \equiv 10^{4} L_{\nu_{\alpha}}$ ). The plots presented show that the maximum on the $\epsilon_{\text {res }} \mathcal{L}_{\nu_{e}}\left(\epsilon_{\text {res }}\right)$ curves occurs at different scaled neutrino momentum values $\epsilon_{\max }$. For each case, the resonance sweeps adiabatically and continuously up to $\epsilon_{\max }$. For $\epsilon_{\text {res }}>\epsilon_{\max }$ the resonance sweeps non-adiabatically resulting in an incomplete conversion $\nu_{\alpha} \rightarrow \nu_{s}$ and a partial depletion of $\mathcal{L}_{\nu_{\alpha}}$.

Figure 2 presents the dependence of the final phase-space distribution of sterile neutrino $f_{\nu_{s}}(\epsilon)$ on the comoving momentum $\epsilon=p / T$ obtained for $\nu_{e} \rightarrow \nu_{s}$ flavor transformation. The models with fixed $m_{s}$ and $\sin ^{2} 2 \theta$ and increasing $L_{4}$ values have increasing average of the comoving momenta $\langle p / T\rangle$, leading to larger-scale cutoffs in the gravitational potential and matter power spectra. The same behavior is present for models with fixed $L_{4}$ and $\sin ^{2} 2 \theta$ and increasing $m_{s}$ values. The models with fixed $L_{4}$ and $m_{s}$ and increasing $\sin ^{2} 2 \theta$ values have opposite behaviors. Larger values of $\sin ^{2} 2 \theta$ lead to larger sterile neutrino production rates. The resonance through momentum distribution occurs in this case at higher temperatures and smaller comoving momenta, as can be seen from Eqs.(2.2).

For this computation we assume a background cosmology consistent with the most recent cosmological measurements [2] with energy densities of $\Omega_{m}=0.28$ in matter, $\Omega_{\Lambda}=0.72$ in cosmological constant, a Hubble parameter of $H_{0}=69 \mathrm{~km} \mathrm{~s}^{-1} \mathrm{Mpc}^{-1}$ and a total active neutrino mass $m_{\nu}=0.35 \mathrm{eV}$, as indicated by the minimal extension of the base $\Lambda$ CDM model by the addition of three degenerated active neutrino flavors (see model M1 below).

\subsection{Effects of DM sterile neutrino resonant production on $N_{e f f}$}

We examine the impact of DM sterile neutrino resonant production on the effective number of relativistic degrees of freedom, $N_{e f f}$, parameter related to the radiation energy density at the photon decoupling. We infer $N_{e f f}$ from the value of $r_{s} / r_{d}$, the ratio of the sound horizon $r_{s}$ to the photon diffusion length $r_{d}$ at the photon decoupling, parameter proposed to be used as a proxy for $N_{e f f}$ in the case of cosmological models that involve non-equilibrium neutrino distributions $[67,68]$.

A simple scaling analysis [67] show that a larger Hubble rate results in a smaller value of the ratio $r_{s} / r_{d}$ and since $\Delta\left(r_{s} / r_{d}\right) \sim-\Delta N_{\text {eff }}\left(r_{s} / r_{d}\right.$ is monotonically decreasing with $\left.N_{e f f}\right)$ this imply that a larger Hubble rate leads to a larger value of $N_{e f f}$.

Employing the same background cosmology as in previous subsection and a primordial helium mass fraction $Y_{P}=0.24$, we compute the evolution with the scale factor of $r_{s}$ and $r_{d}$ given by the following integrals:

$$
r_{s}(a)=\int_{0}^{a} \frac{\mathrm{d} a^{\prime}}{a^{\prime 2} H\left(a^{\prime}\right)} \frac{1}{3(1+R)}, \quad r_{d}^{2}(a)=\pi^{2} \int_{0}^{a} \frac{\mathrm{d} a^{\prime}}{a^{\prime 2} H\left(a^{\prime}\right)} \frac{1}{a n_{e}\left(a^{\prime}\right) \sigma_{T}} \frac{R^{2}+\frac{16}{15}(1+R)}{6(1+R)^{2}}(2 .
$$



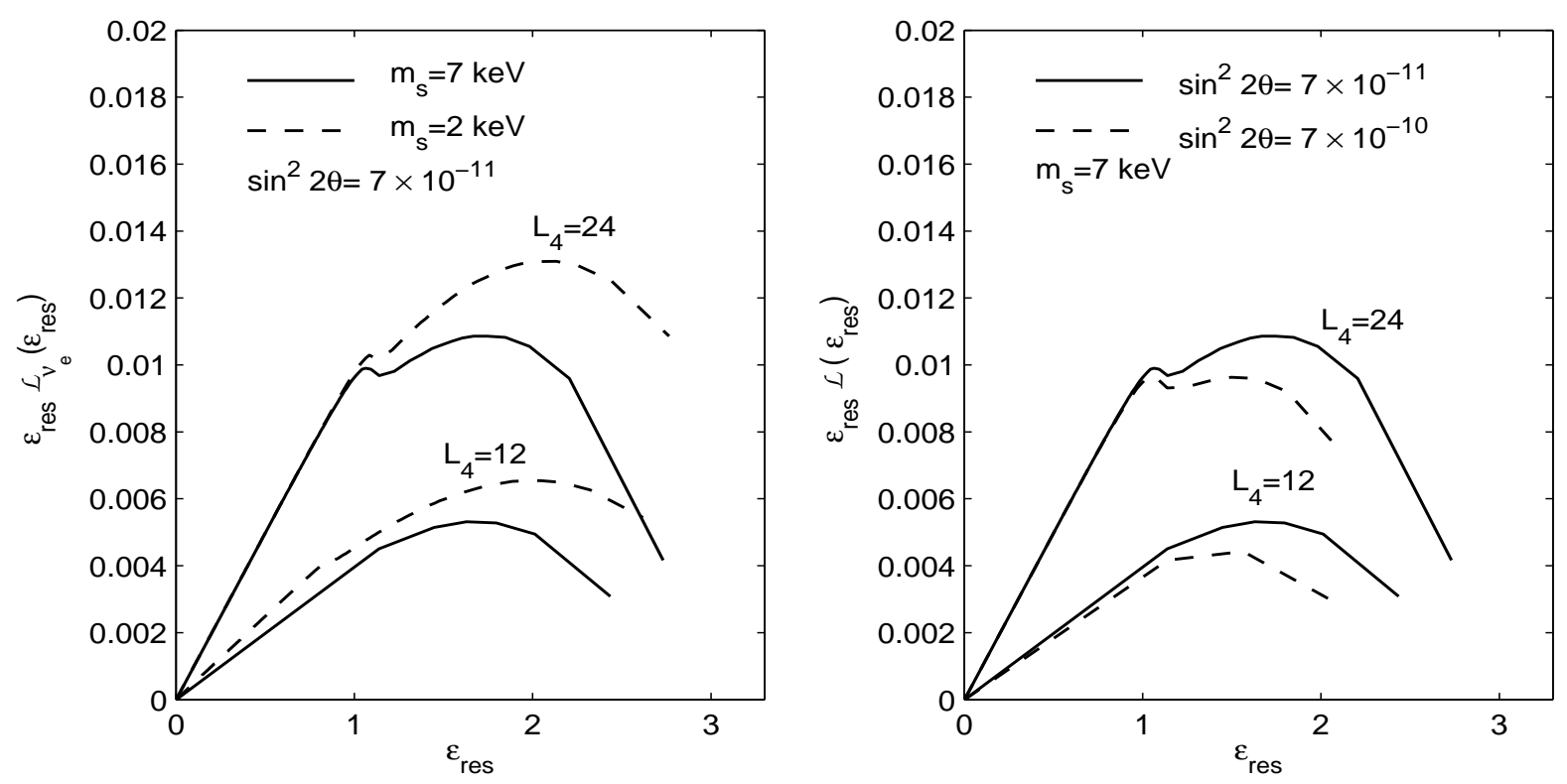

Figure 1. Left: The dependence of $\epsilon_{\text {res }} \mathcal{L}_{\nu_{e}}\left(\epsilon_{\text {res }}\right)$ on sterile neutrino mass $m_{s}=7 \mathrm{keV}$ (continuous line) and $m_{s}=2 \mathrm{keV}$ (dashed line) for initial lepton numbers $L_{4}=12,24$ and sterile neutrino mixing angle $\sin ^{2} 2 \theta=7 \times 10^{-11}$. Right: The dependence of $\epsilon_{\text {res }} \mathcal{L}_{\nu_{e}}\left(\epsilon_{\text {res }}\right)$ on sterile neutrino mixing angle $\sin ^{2} 2 \theta=7 \times 10^{-11}$ (continuous line) and $\sin ^{2} 2 \theta=7 \times 10^{-10}$ (dashed line) for initial lepton numbers $L_{4}=12,24$ and sterile neutrino mass $m_{s}=7 \mathrm{keV}$. We define $L_{4} \equiv 10^{4} L_{\nu_{\alpha}}$.

where $H\left(a^{\prime}\right)$ is the Hubble expansion rate, $n_{e}\left(a^{\prime}\right)$ is the free-electron number density, $\sigma_{T}$ is the Thomson cross section and $R(a) \equiv 3 \rho_{b} /\left(4 \rho_{\gamma}\right)$ is the ratio of the baryon energy density $\rho_{b}$, to the photon energy density $\rho_{\gamma}$. We also use the recombination code RECFAST [80] to evolve the free electron fraction $X_{e}$ and the number density of free electrons $n_{e}$ with the scale factor, required for the computation of $r_{d}$.

In Figure 3 we plot the evolution with the scale factor of the changes in $N_{e f f}$ and $X_{e}$ relative to the model with zero lepton number for sterile neutrino resonant production models with $L_{4}=4.3$ and 7.5 (RP-case) and for the leptonic asymmetric models with the same values of $L_{4}$ ( $L_{\nu}$-only case). We see a larger value of $N_{\text {eff }}$ at the photon decoupling (that imply a higher Hubble expansion rate) in both cases, relative to the case with zero lepton number. The increase of $N_{\text {eff }}$ is smaller in the RP case relative to the $L_{\nu}$-only case with the same of lepton number.

Also, a larger Hubble expansion rate at the photon decoupling implies a larger value of the free-electron fraction $X_{e}$ and an earlier epoch of $X_{e}$ freeze-out.

\section{Cosmological constraints}

\subsection{Method and datasets}

We compute the angular power spectra of the CMB anisotropies, the matter density fluctuations power spectrum and the gravitational lensing potential power spectrum with the Boltzmann Code for Anisotropies in the Microwave Background, CAMB [81], modified to allow the resonant flavor conversion production calculations as presented in the previous 

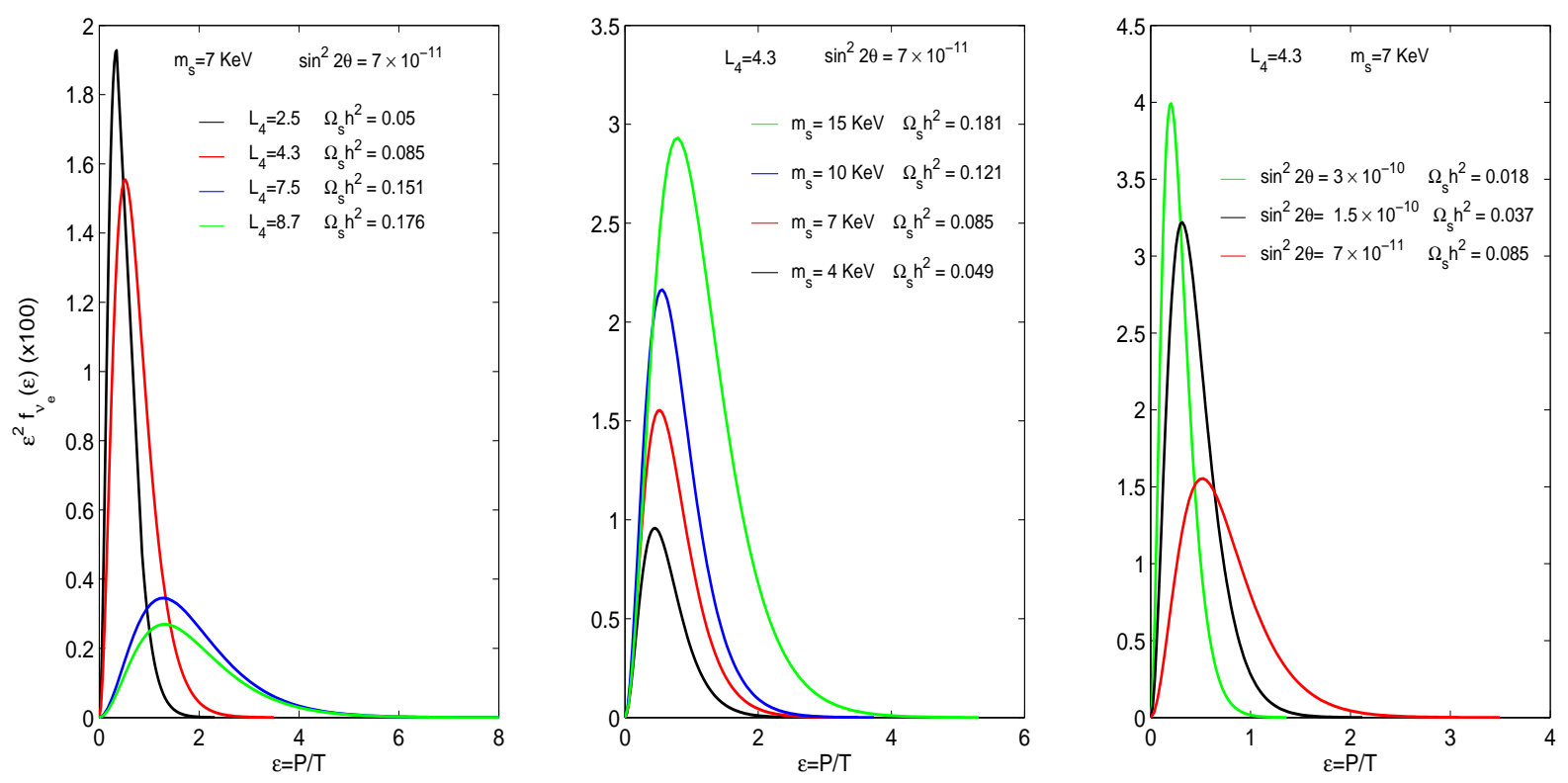

Figure 2. The dependence of the final phase-space distribution of sterile neutrino $f_{\nu_{s}}$ on the comoving momentum $\epsilon=p / T$ obtained for $\nu_{e} \rightarrow \nu_{s}$ flavor transformation. From top to bootom the plots represent sterile neutrino phase-space distributions of: $m_{s}=7 \mathrm{keV}$ and $\sin ^{2} 2 \theta=7 \times 10^{-11}$ models with $L_{4}=2.5,4.3,7.5$ and 8.7 (left panel), $L_{4}=4.3$ and $\sin ^{2} 2 \theta=7 \times 10^{-11}$ models with $m_{s}=4,7,10$ and $15 \mathrm{keV}$ (middle panel) and $L_{4}=4.3$ and $m_{s}=7 \mathrm{keV}$ models with $\sin ^{2} 2 \theta=3 \times 10^{-10}, 1.5 \times 10^{-10}$ and $7 \times 10^{-11}$. For each case we also indicate the sterile neutrino energy density parameter, $\Omega_{s} \mathrm{~h}^{2}$ at the present time.

section. The $\nu_{\alpha} / \bar{\nu}_{\alpha}$ and $\nu_{s}$ phase-space distributions alter the homogeneous $\nu_{\alpha} / \bar{\nu}_{\alpha}$ and $\nu_{s}$ energy density and pressure, the perturbed inhomogeneous components and the gravitational source term in the Boltzmann equation that depends on the logarithmic derivative of the phase-space distributions with respect to comoving momentum.

The impact of the leptonic asymmetry on cosmological observables has been discussed in the literature [82-84]. In particular, the non-zero leptonic asymmetry change the neutrino velocity dispersion, the neutrino free-streaming length and the Jeans mass [85, 86]. The leptonic asymmetry also increases the radiation energy density, usually parametrized by variation of the number of relativistic degrees of freedom $\Delta N_{e f f}\left(\xi_{\nu}\right)$ given by:

$$
\Delta N_{\text {eff }}\left(\xi_{\nu}\right)=3\left[\frac{30}{7}\left(\frac{\xi_{\nu}}{\pi}\right)^{2}+\frac{15}{7}\left(\frac{\xi_{\nu}}{\pi}\right)^{4}\right] .
$$

Figure 4 presents the gravitational lensing potential power spectrum $C_{l}^{\phi, \phi}$ and the matter density fluctuations power spectrum $P(k)$ for $m_{s}=7.14 \mathrm{keV}, \sin ^{2} 2 \theta=6.46 \times 10^{-11}$ and lepton asymmetry numbers $L_{4}=2.5,4.3$ and 7.5 , having larger cutoff scales.

The leptonic asymmetry shifts the beta equilibrium between protons and neutrons at the BBN epoch, leading to indirect effects on the CMB anisotropy through the primordial helium mass fraction $Y_{P}$ that decreases monotonically with increasing $\xi_{e}$.

The primordial helium mass fraction is also sensitive to the shape of $\nu_{e} / \bar{\nu}_{e}$ phase-space distribution. As the $\nu_{e} / \bar{\nu}_{e}$ phase-space distributions determine the rates of the neutron and 

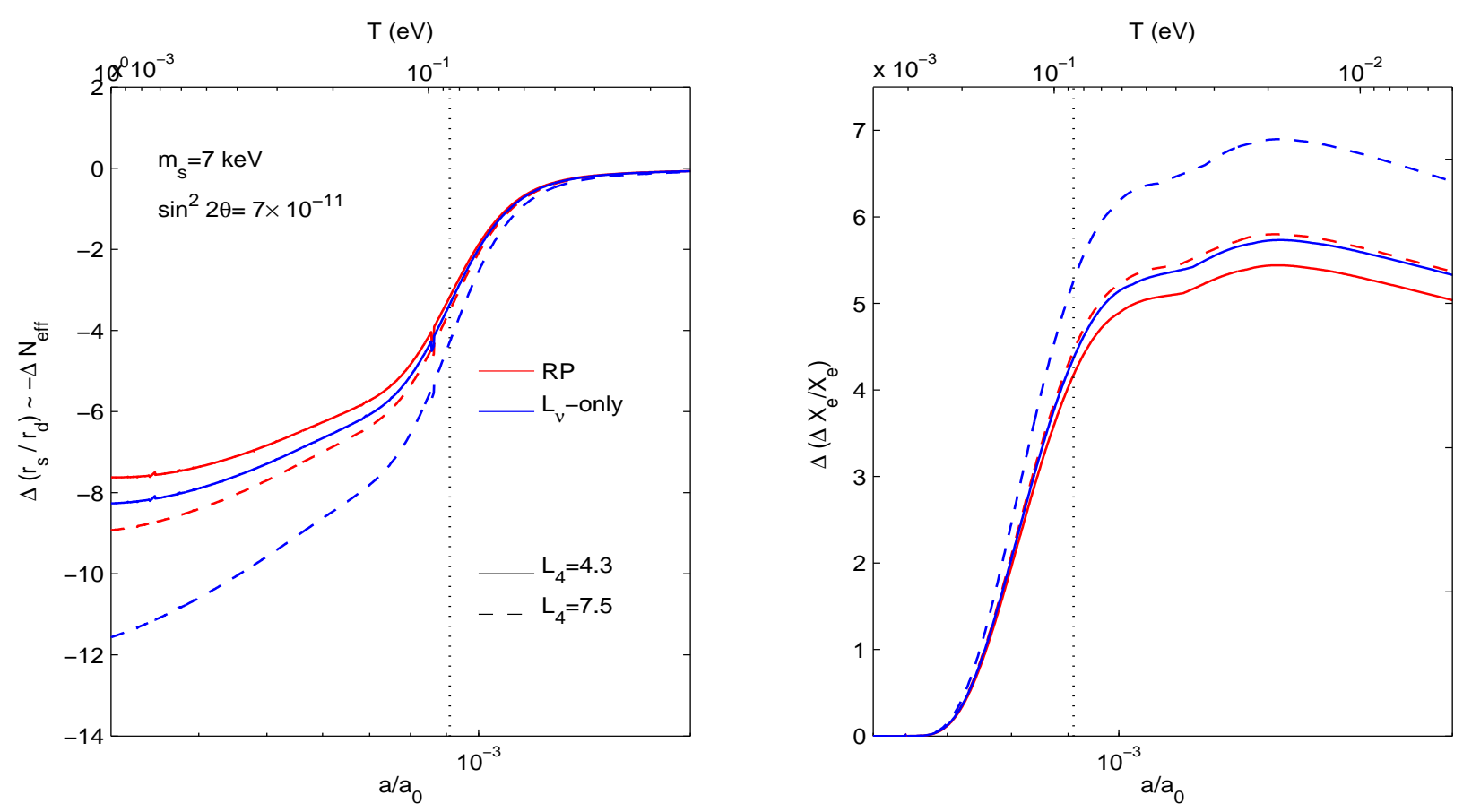

Figure 3. Evolution with the scale factor of the changes in the effective number of relativistic degrees of freedom, $N_{e f f}$, (left) in and of the free electron fraction, $X_{e}$, (right) for two lepton number values, $L_{4}=4.3$ (continuous lines) and $L_{4}=7.5$ (dashed lines), relative to the case with zero lepton number, for sterile neutrino resonant production models (red lines - RP) and and leptonic asymmetric models (blue lines - $L_{\nu}$ only). The vertical dotted line corresponds to the epoch of photon decoupling $\left(a_{d c} \simeq 9 \times 10^{-4}\right)$.

proton interaction rates at $\mathrm{BBN}$, the $\nu_{e} / \bar{\nu}_{e}$ spectra will change these rates and hence the helium mass fraction over the case with thermal Fermi-Dirac spectrum [66, 69, 70]. We use the BBN PArthENoPE code $[87,88]$ to compute the helium abundance, $Y_{P}$, for different values of $\Omega_{b} h^{2}, \Delta N_{e f f}, \xi_{\nu}$ and changes of neutron and proton interaction rates.

We split the total variation of the radiation energy density in two uncorrelated contributions:

$$
\Delta N_{e f f}=\Delta N_{e f f}\left(\xi_{\nu}\right)+\Delta N_{e f f}^{o t h},
$$

first due to the net leptonic asymmetry of the neutrino background as given by Eq. (3.1) and second due to possible extra contributions from unknown processes.

We adapt the latest version of the publicly available package CosmoMC [89] for our cosmological analysis and use the following datasets and likelihood codes:

- The Planck CMB anisotropy angular power spectrum, combined with WMAP-9 year polarization power spectrum at low $\ell$ [90] and the corresponding codes [1, 91]: Commander, that computes the low- $l$ PLANCK likelihood, CamSpec, that computes the PlancK likelihood for $50 \leq l \leq 2500$, LowLike, that computes the likelihoods for $2 \leq l \leq 32$ polarization data and Lensing, that computes the likelihoods from PlANCK lensing power spectrum for $40 \leq l \leq 400$ [92].

- The high-l CMB data from Atacama Cosmology Telescope(ACT) $[93,94]$ and the South Pole Telescope (SPT) [95, 96]. 

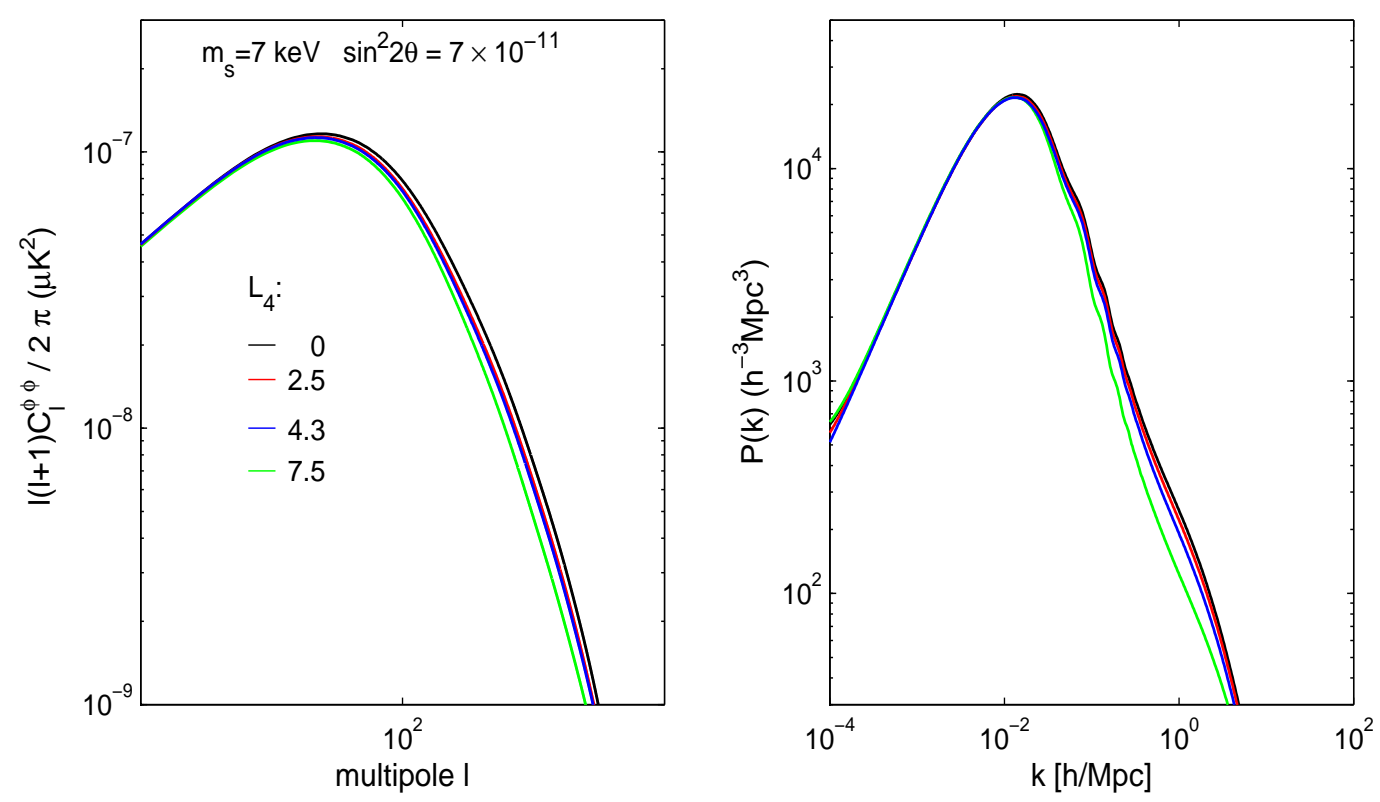

Figure 4. The reconstructed gravitational lensing potential power spectrum $C_{l}^{\phi, \phi}$ (left) and the matter density fluctuations power spectrum $P(k)$ (right) for $m_{s}=7.14 \mathrm{keV}, \sin ^{2} 2 \theta=6.46 \times 10^{-11}$ and lepton asymmetry numbers $L_{4}=2.5,4.3$ and 7.5 having larger cutoff scales.

- The baryon acoustic oscillation (BAO) from the Sloan Digital Sky Survey (SDSS) Data Release 7 (DR7) [97] at redshifts $z=0.2,0.35$, the reanalyzed SDSS DR7 galaxy catalog data at $\mathrm{z}=0.35[98,99]$, the SDSS Baryon Oscillation Spectroscopic Survey(BOSS) Data Release 9 (DR9) at $\mathrm{z}=0.57[100,101]$ and the 6dF Galaxy Survey (6dFGS) [102] $\mathrm{BAO}$ data at $\mathrm{z}=0.1[102,103]$.

\subsection{Analysis}

In this section we evaluate the impact of sterile neutrino resonant production mechanism on the cosmological parameters. We consider the following extensions of the standard $\Lambda \mathrm{CDM}$ cosmological model:

- M1: $\Lambda \mathrm{CDM}+\Sigma m_{\nu_{\alpha}}$ :

The minimal extension of the base $\Lambda$ CDM model by the addition of three degenerated species of massive neutrinos with total mass $\Sigma m_{\nu_{\alpha}}$ (within which $\Lambda$ CDM is nested at $N_{e f f}=3.046$ and $\left.Y_{P}=0.24\right)$.

- M2: $\Lambda \mathrm{CDM}+\Sigma m_{\nu_{\alpha}}+\Sigma \xi_{\nu_{\alpha}}+\Delta N_{e f f}+Y_{P}$ :

The addition to $\mathbf{M} 1$ of the neutrino chemical potential $\Sigma \xi_{\nu_{\alpha}}$ the extra radiation energy density $\Delta N_{\text {eff }}$ as given in Eq. (3.2) and the BBN prediction of the primordial helium abundance $Y_{P}$.

- M3: $\Lambda$ CDM+ $+\Sigma m_{\nu_{\alpha}}+\Sigma \xi_{\nu_{\alpha}}+\Delta N_{e f f}+m_{s}+\sin ^{2} 2 \theta+Y_{P}$ :

The addition to M2 of one DM sterile neutrino with the mass $m_{s}$, the matter mixing angle $\sin ^{2} 2 \theta$ and the BBN prediction of the primordial helium abundance $Y_{P}$.

Our main results are summarized in Table 1. 
Table 1. The table shows the mean values and the absolute errors on the main cosmological parameters obtained from the fits of different extensions of the $\Lambda$ CDM model discussed in the text with $\mathrm{PLANCK}+\mathrm{WP}+$ highL+BAO+lensing dataset. For all parameters, except $\Sigma m_{\nu}$ and $\sin ^{2} 2 \theta$ we quote the errors at $68 \%$ CL. For $\Sigma m_{\nu}$ and $\sin ^{2} 2 \theta$ we give the values of $95 \%$ upper limits.

\begin{tabular}{lccc}
\hline \hline Parameter & M1 & M2 & M3 \\
\hline$\Omega_{b} h^{2}$ & $0.02227 \pm 0.00019$ & $0.02221 \pm 0.00024$ & $0.02209 \pm 0.00023$ \\
$\Omega_{c d m} h^{2}$ & $0.1161 \pm 0.0015$ & $0.1162 \pm 0.0015$ & $0.1169 \pm 0.0021$ \\
$100 \theta_{M C}$ & $1.04156 \pm 0.00059$ & $1.04222 \pm 0.00095$ & $1.04261 \pm 0.00072$ \\
$\tau$ & $0.0882 \pm 0.0091$ & $0.0851 \pm 0.0092$ & $0.0814 \pm 0.0121$ \\
$\Sigma m_{\nu_{\alpha}}(\mathrm{eV})$ & $<0.35$ & $<0.25$ & $<0.21$ \\
$m_{s}(\mathrm{keV})$ & - & - & $6.08 \pm 1.62$ \\
$\sin ^{2} 2 \theta$ & - & - & $<5.61 \times 10^{-10}$ \\
$\xi_{\nu_{\alpha}}$ & - & $-0.062 \pm 0.079$ & $0.000002 \pm 0.000093$ \\
$N_{e f f}$ & - & $3.37 \pm 0.19$ & $3.25 \pm 0.15$ \\
$n_{s}$ & $0.9586 \pm 0.0046$ & $0.9679 \pm 0.0048$ & $0.9711 \pm 0.0052$ \\
$\ln 10^{10} A_{s}$ & $3.213 \pm 0.019$ & $3.231 \pm 0.023$ & $3.192 \pm 0.041$ \\
\hline$\Omega_{\Lambda}$ & $0.699 \pm 0.012$ & $0.712 \pm 0.011$ & $0.713 \pm 0.012$ \\
$\Omega_{m}$ & $0.302 \pm 0.012$ & $0.288 \pm 0.011$ & $0.287 \pm 0.011$ \\
$\sigma_{8}$ & $0.793 \pm 0.021$ & $0.801 \pm 0.022$ & $0.772 \pm 0.015$ \\
$H_{0}$ & $68.16 \pm 1.02$ & $70.15 \pm 1.36$ & $69.95 \pm 1.17$ \\
$Y_{p}$ & 0.24 & $0.267 \pm 0.019$ & $0.24968 \pm 0.00011$ \\
$z_{e q}$ & $3306 \pm 33$ & $3221 \pm 36$ & $3183 \pm 46$ \\
$L_{\nu_{\alpha}}$ & - & $-0.015 \pm 0.012$ & $0.000123 \pm 0.000022$ \\
$r_{s} / r_{d}$ & $6.478 \pm 0.011$ & $6.389 \pm 0.032$ & $7.013 \pm 0.011$ \\
\hline \hline
\end{tabular}

\subsubsection{Neutrino masses and mixing}

Top left panel from Figure 5 presents the likelihood posterior distributions of total active neutrino mass, $\Sigma m_{\nu_{\alpha}}$, showing a decrease of the upper limit (at $95 \% \mathrm{CL}$ ), from $0.35 \mathrm{eV}$, for the case of minimal extension of the $\Lambda \mathrm{CDM}$ model (M1), to $0.25 \mathrm{eV}$ when only the leptonic asymmetry is included (M2) and a subsequent decrease to $0.21 \mathrm{eV}$ for the RP sterile neutrino scenario (M3).

In the top right panel of Figure 5 we plot the likelihood posterior probability distribution of the sterile neutrino mass fraction $f_{\nu_{s}}=\Omega_{s} h^{2} / \Omega_{m} h^{2}$. The dominant effect on sterile neutrino resonant production is given by the value of the initial potential lepton number, $\mathcal{L}_{\nu}^{\text {init }}$, rather than sterile neutrino mass. This is shown in the bottom panels from Figure 5 that present the joint confidence regions $\mathcal{L}_{\nu_{\alpha}}^{\text {init }}-f_{\nu_{s}}$ and $m_{s}-f_{\nu_{s}}$. We find $f_{\nu_{s}}<0.078$ for a value of lepton number per flavor $L_{4}=1.23 \pm 0.04$, sterile neutrino mass $m_{s}=6.08 \pm 3.22 \mathrm{keV}$ and mixing angle $\sin ^{2} 2 \theta<5.61 \times 10^{-10}$ (errors at $95 \% \mathrm{CL}$ ).

Figure 6 presents the marginalized likelihood posterior distribution obtained for $m_{s}$ and the joint confidence regions $m_{s}-\sin ^{2} 2 \theta$.

\subsection{2 $\quad{ }^{4} \mathrm{He}$ abundance yield}

The BBN ${ }^{4} \mathrm{He}$ yield depends sensitively $\nu_{e} / \bar{\nu}_{e}$ degeneracy parameter, $\xi_{\nu_{e}}$, as well as on the shape of its phase-space distribution function. We find the preference of cosmological data for smaller values of $\xi_{\nu_{e}}$ and ${ }^{4} \mathrm{He}$ mass fraction, $Y_{P}$, in the case of RP sterile neutrino scenario 

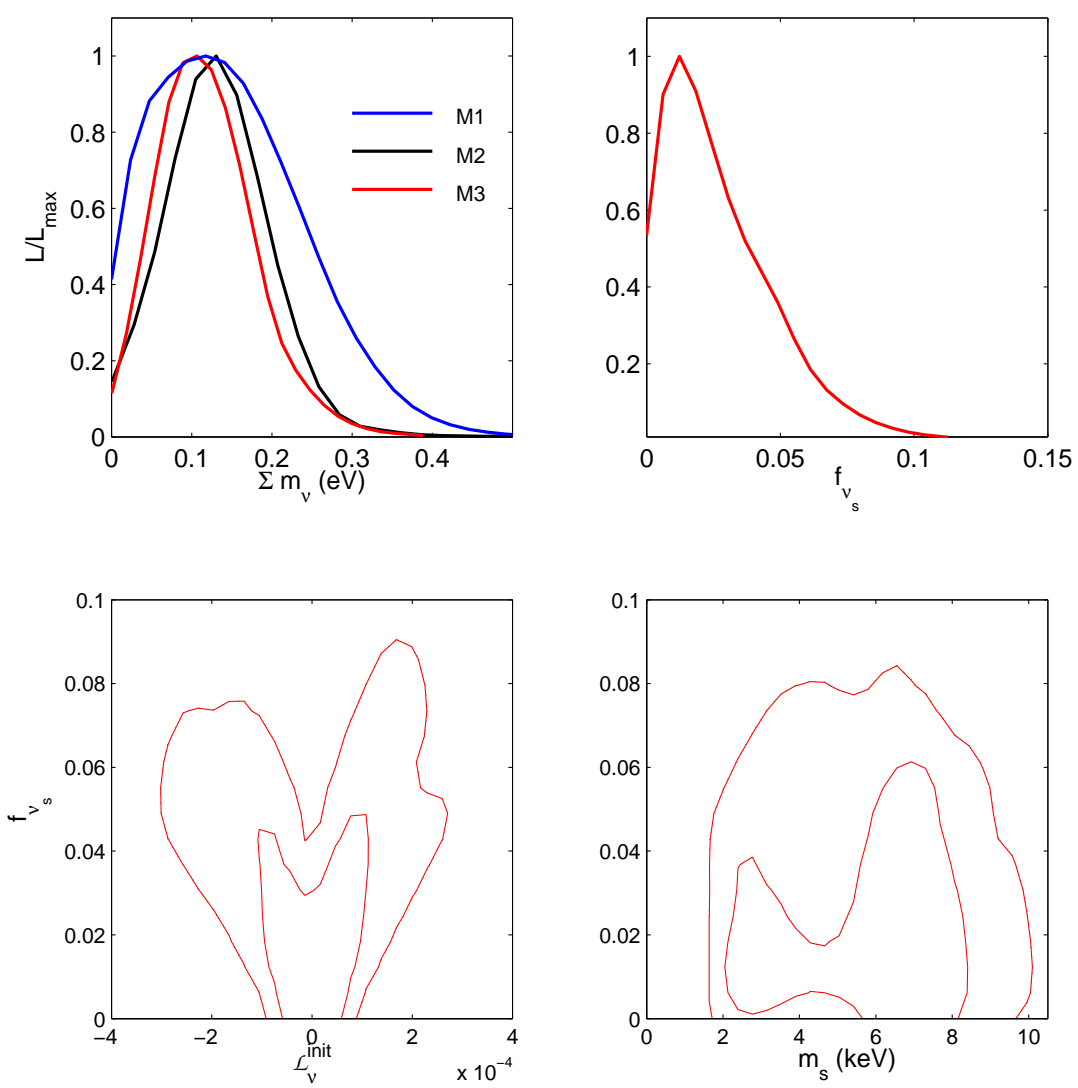

Figure 5. Top: The marginalized likelihood posterior distributions for active neutrinos total mass $\Sigma m_{\nu_{\alpha}}$ and and sterile neutrino mass fraction, $f_{\nu_{s}}=\Omega_{s} h^{2} / \Omega_{m} h^{2}$, as obtained from the fits of different extensions of the $\Lambda \mathrm{CDM}$ cosmological model with PLANCK $+\mathrm{WP}+$ highL+BAO+lensing dataset (see also the text). Bottom: The joint confidence regions $\mathcal{L}_{\nu_{\alpha}}^{\text {init }}-f_{\nu_{s}}$ and $m_{s}-f_{\nu_{s}}$ (at $68 \%$ CL and $95 \%$ CL).

(M3) when compared to the similar predictions obtained in the model with leptonic asymmetry only (M2). This is shown in Figure 7 where we plot the marginalized likelihood probability distribution of $\xi_{\nu_{e}}$ obtained from the fit of model M3 and the joint confidence regions $\xi_{\nu_{e}}$ $Y_{P}$ from the fit of model M2 and M3 with our dataset. We obtain $\xi_{\nu}=0.0005 \pm 0.0004$ and $Y_{P}=0.2497 \pm 0.0002(95 \% \mathrm{CL})$ for M3 case, in very good agreement with the PLANCK determination [2], $Y_{P}=0.2485 \pm 0.0002$, and the latest observational bounds $Y_{P}=0.24968 \pm 0.00022$ [104].

\section{Conclusions}

Recently, few independent detections of a weak X-ray emission line at an energy of $\sim 3.5 \mathrm{keV}$ seen toward a number of astrophysical sites have been reported. Until now, none of these searches were able to establish precisely the origin of this line. If confirmed, this signal could be the signature of decaying DM sterile neutrino with a mass of $\sim 7.1 \mathrm{keV}$.

The DM sterile neutrino production via resonant MSW conversion of active neutrinos to sterile neutrinos through the Shi-Fuller mechanism [38] is the simplest model for the DM origin of the undefined $\sim 3.5 \mathrm{keV}$ of X-ray line. 

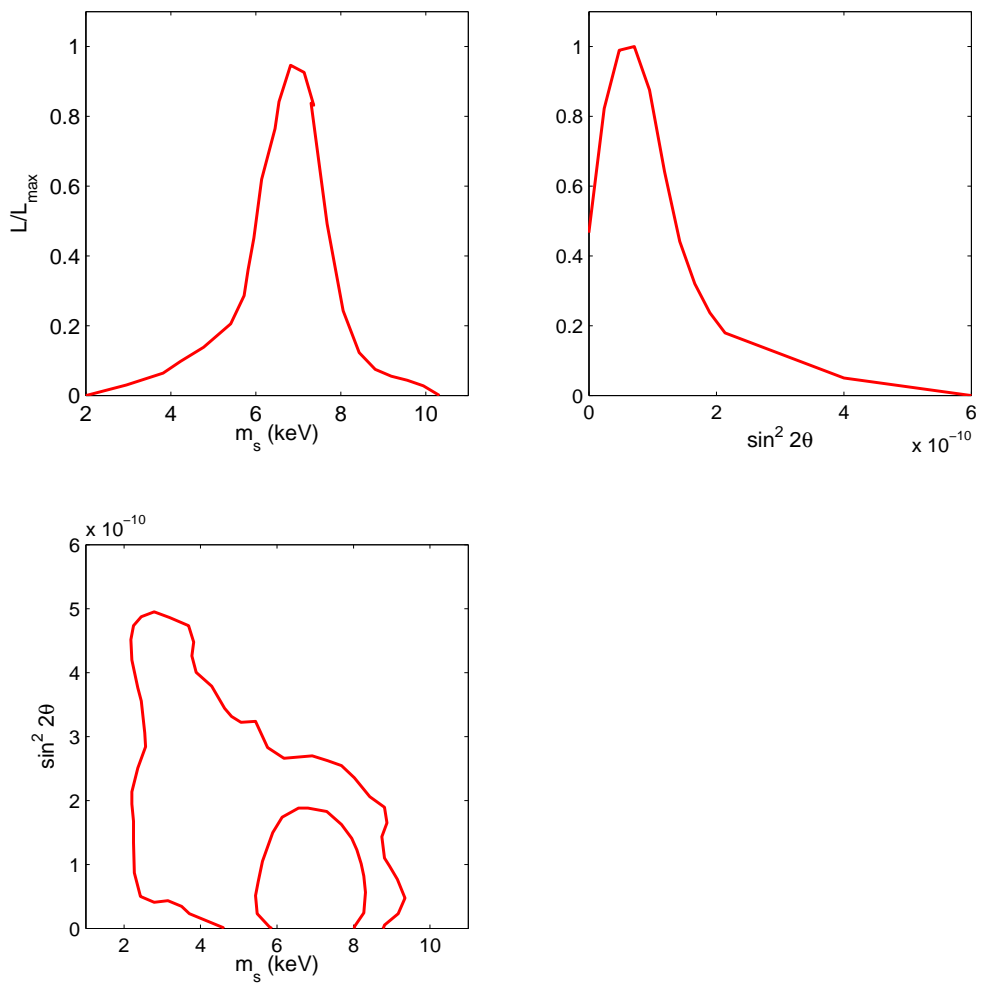

Figure 6. Top: The marginalized likelihood posterior distributions obtained for the sterile neutrino mass, $m_{s}$ and the mixing angle $\sin ^{2} 2 \theta$. Bottom: The joint confidence regions $m_{s}-\sin ^{2} 2 \theta$ (at $68 \%$ CL and $95 \% \mathrm{CL})$.
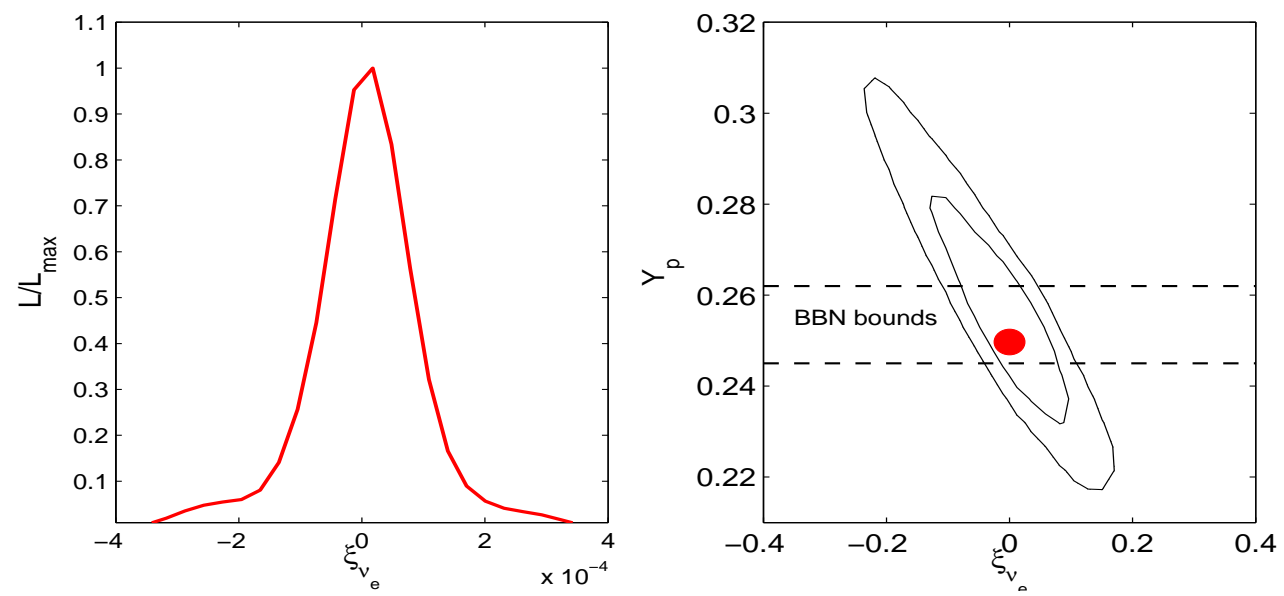

Figure 7. Left: The marginalized likelihood probability distribution of $\xi_{\nu_{e}}$ from the fit of model M3 with PLANCK+WP+highL+BAO+lensing dataset. Right: The joint confidence regions $\xi_{\nu_{e}}-Y_{P}$ (at $68 \%$ CL and 95\% CL) from the fit of model M2 (black) and M3 (red) with the same dataset. The latest observational bounds [104] are also plotted (at 68\%CL). 


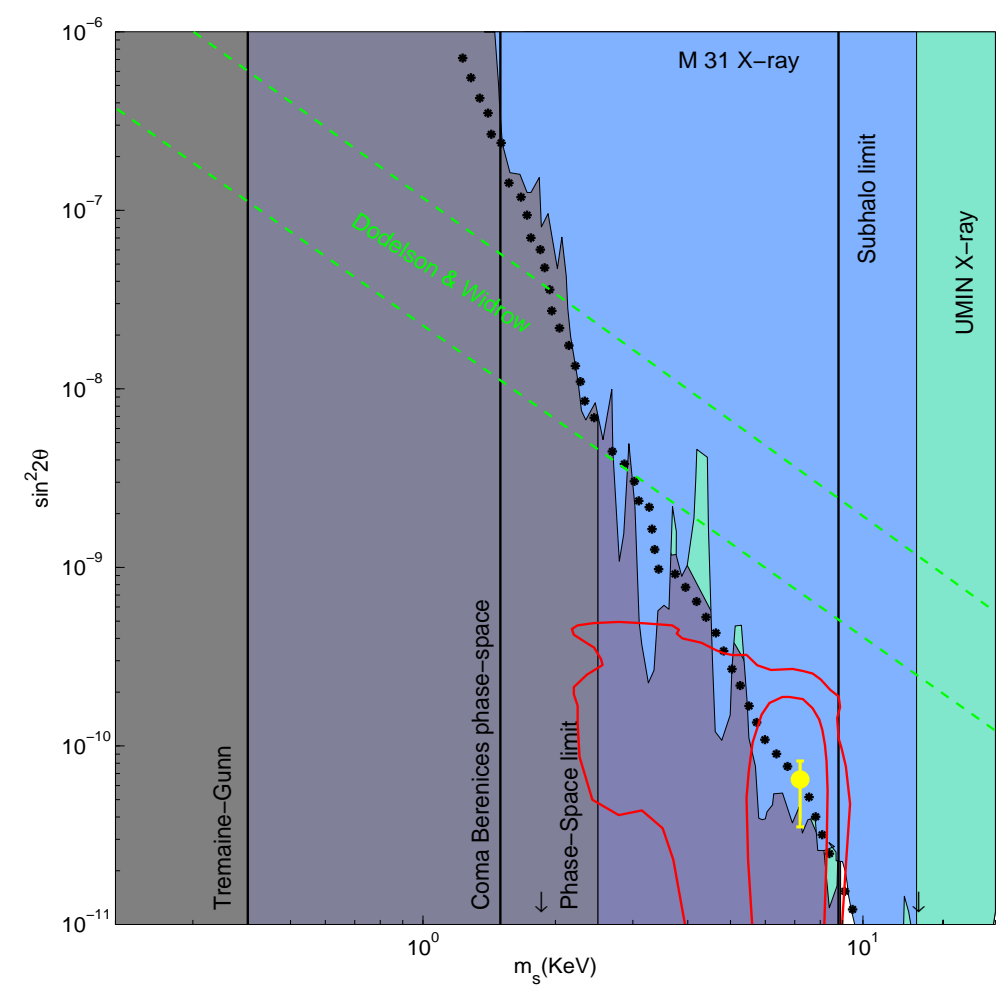

Figure 8. Two-dimensional marginalized joint probability distributions at $68 \%$ and $95 \%$ CL (red lines) showing the dependence of RP sterile neutrino mass, $m_{s}$, and the matter mixing angle, $\sin ^{2} 2 \theta$, obtained from our cosmological analysis. We also show the 95\% CL upper limits reproduced from Ref.[32] based on deep Chandra [32] and XMM-Newton [35] observations of M31 and Suzuku observations of Ursa Minor [55, 56]. The vertical lines represents the lower mass limits from Tremaine-Gunn phase-space considerations $\left(m_{s} \sim 0.4\right) \mathrm{keV}$ [27], Coma Berenices phase-space $\left(m_{s}^{\mathrm{DW}} \sim 1.5\right) \mathrm{keV}$, Segue I phase-space $\left(m_{s}^{\mathrm{DW}} \sim 2.5\right) \mathrm{keV}$ and M31 subhalo counts $\left(m_{s}^{\mathrm{DW}} \sim 8.8\right) \mathrm{keV}$. The upper and lower bounds (95\% CL) of sterile neutrino mass obtained in Dodelson-Widrow model [23] are also indicated (green dashed lines). The yellow symbol corresponds to the detection from Ref.[50] $\left(m_{s} \simeq 7.1 \mathrm{keV}\right.$, $\sin ^{2} 2 \theta=7 \times 10^{-11}$ and $90 \%$ statistical error bar).

The RP sterile neutrino parameters required to produce this signal have been recently inferred from the linear large scale structure constraints to produce full DM density [62]. These parameters are consistent with the Local Group and high-z galaxy count constraints, fulfilling previously determined requirements to successfully solve "missing satellite problem" and "too-big-to-fail problem". The cosmological constraints on subdominant RP sterile neutrino parameters have not yet derived.

In this paper we place constraints on RP sterile neutrino parameters in a $\Lambda$ CWDM model containing a mixture of WDM in the form of RP sterile neutrino and CDM, by using most of the present cosmological measurements.

We make a coupled treatment of the weak decoupling, primordial nucleosynthesis and photon decoupling epochs in the sterile neutrino RP scenario including the extra radiation energy density parametrized by the effective number of relativistic degrees of freedom $N_{\text {eff }}$.

We compute the radiation and matter perturbations including the full resonance sweep solution for $\nu_{\alpha} / \bar{\nu}_{\alpha} \rightarrow \nu_{s}$ flavor conversion in the expanding Universe and provide constraints 
on the cosmological parameters and sterile neutrino properties, by using most of the present cosmological measurements. In particular, the high precision CMB temperature anisotropy and the reconstructed CMB gravitational lensing potential power spectra obtained by the PLANCK satellite have impact on cosmological parameter degeneracies when DM sterile neutrino scenario is considered.

We find that the values of the main cosmological parameters are in agreement with the predictions of the minimal extension of the base $\Lambda$ CDM model except for the active neutrino total mass that is decreased to $\Sigma m_{\nu_{\alpha}}<0.21 \mathrm{eV}$ (at 95\% CL).

Figure 8 presents the $m_{s}-\sin ^{2} 2 \theta$ joint probability distributions obtained from our cosmological analysis, compared with the most of the existing similar constrains. The cosmological measurements are in agreement with the sterile neutrino RP scenario with the following parameters (errors at 95\% CL): $m_{s}=6.28 \pm 3.2 \mathrm{keV}$, mixing angle $\sin ^{2} 2 \theta<5.61 \times 10^{-10}$, lepton number per flavor $L_{4}=1.23 \pm 0.04$ and sterile neutrino mass fraction $f_{\nu_{s}}<0.078$.

Our results are in agreement with the sterile neutrino resonant production parameters inferred in Ref. [62] from the linear large scale structure constraints to produce full Dark Matter density.

This reflects the sensitivity of the high precision CMB observables to the physics that determines the neutrino energy spectra at different epochs, that require a self-consistent and coupled treatment [68].

\section{Acknowledgments}

This work was supported by a grant of the Ministery of National Education, CNCS-UEFISCDI, project number PN-II-ID-PCE-2012-4-0511.

\section{References}

[1] Planck Collaboration; P. A. R. Ade et al., Planck 2013 results. I. Overview of products and scientific results, Astron. ES Astrophys. 571 (2014) 48 [arXiv:1303.5062].

[2] Planck Collaboration; P. A. R. Ade, et al., Planck 2013 results. XVI.Cosmological Parameters, Astron. \& Astrophys. 571 (2014) 66 [arXiv:1303.5076].

[3] A. Klypin, A. V. Kravtsov, O. Valenzuela, F. Prada, Where Are the Missing Galactic Satellites?, Astrophys. J 522 (1999) 82 [arXiv:astro-ph/9901240].

[4] A. Kravtsov Dark Matter Substructure and Dwarf Galactic Satellites, Adv. in Astron. 281913 (2010) [arXiv:0906.3295].

[5] M. A. Zwaan, M. J. Meyer, L. Staveley-Smith, The velocity function of gas-rich galaxies, Mon. Not. Roy. Astron. Soc. 403 (2010) 1969 [arXiv:0912.1754].

[6] E. Papastergis, A. M. Martin, R. Giovanelli, M. P. Haynes, The Velocity Width Function of Galaxies from the $40 \%$ ALFALFA Survey: Shedding Light on the Cold Dark Matter Overabundance Problem, Astrophys. J. 739 (2011) 38 [arXiv:1106.0710].

[7] P. Salucci, A. Burkert, Dark Matter Scaling Relations, Astrophys. J. 737 (2000) 9 [arXiv:astro-ph/0004397].

[8] G. Gentile, P. Salucci, U. Klein, D. Vergani, P. Kalberla, The cored distribution of dark matter in spiral galaxies, Mon. Not. Roy. Astron. Soc. 351 (2004) 903 [arXiv:astro-ph/0403154].

[9] R. Kuzio de Naray, T. Kaufmann, Recovering cores and cusps in dark matter haloes using mock velocity field observations, Mon. Not. Roy. Astron. Soc. 414 (2011) 3617 [arXiv:1012.3471]. 
[10] M. Boylan-Kolchin, J. S. Bullock, M. Kaplinghat, Too big to fail? The puzzling darkness of massive Milky Way subhaloes Mon. Not. Roy. Astron. Soc. 415 (2011) L40 [arXiv:1103.0007].

[11] M. Boylan-Kolchin, J. S. Bullock, M. Kaplinghat, The Milky Way's bright satellites as an apparent failure of ACDM Mon. Not. Roy. Astron. Soc. 422 (2012) 1203 [arXiv:1111.2048].

[12] F. Governato, C. Brook, L. Mayer, A. Brooks et al., Bulgeless dwarf galaxies and dark matter cores from supernova-driven outflows, Nature 463 (2010) 203 [arXiv:0911.2237].

[13] M. G. Walker, J. Peñarrubia, A Method for Measuring (Slopes of) the Mass Profiles of Dwarf Spheroidal Galaxies, Astrophys. J. 742 (2011) 20 [arXiv:1108.2404].

[14] I. Ferrero, M. G. Abadi, J. F. Navarro, L. V. Sales, S. Gurovich, he dark matter haloes of dwarf galaxies: a challenge for the cold dark matter paradigm?, Mon. Not. Roy. Astron. Soc. 425 (2012) 2817 [arXiv:1111.6609].

[15] S. Garrison-Kimmel, M. Boylan-Kolchin, J. S. Bullock, E. N. Kirby, Too big to fail in the Local Group, Mon. Not. Roy. Astron. Soc. 444 (2014) 222 [arXiv:1404.5313].

[16] G. Jungman, M. Kamionkowski, K. Griest, Supersymmetric dark matter, Phys. Rept. 267 (1996) 195 [arXiv:hep-ph/9506380].

[17] G. Bertone, D. Hooper, J. Silk, Particle dark matter: evidence, candidates and constraints, Phys. Rept. 405 (2005) 279 [arXiv:hep-ph/0404175].

[18] J. L. Feng, Dark Matter Candidates from Particle Physics and Methods of Detection, Ann. Rev. Astron. Astrophys. 48 (2010) 495 [arXiv:1003.0904].

[19] K. Abazajian, G.M.Fuller, M. Patel, Sterile neutrino hot, warm, and cold dark matter, Phys. Rev. D 64 (2001) 3501 [arXiv:astro-ph/0101524].

[20] K.N. Abazajian, G. M. Fuller, Bulk QCD thermodynamics and sterile neutrino dark matter, Phys. Rev. D 66 (2002) 023526.

[21] A. Boyarsky, O. Ruchayskiy, M. Shaposhnikov, The Role of Sterile Neutrinos in Cosmology and Astrophysics, Ann. Rev. Nucl. Part. Sci. 59 (2009) 191 [arXiv:0901.0011].

[22] A. Kusenko, Sterile neutrinos: The dark side of the light fermions, Phys. Rept. 481 (2009) 1 [arXiv:0906.2968].

[23] T. Asaka, S. Blanchet, M. Shaposhnikov, The $\nu M S M$, dark matter and neutrino masses, Phys. Lett B 631 (2005) 151 [arXiv:hep-ph/0503065].

[24] T. Asaka, M. Shaposhnikov, The $\nu M S M$, dark matter and baryon asymmetry of the universe, Phys. Lett 620 (2005) 17 [arXiv:hep-ph/0505013].

[25] A. Boyarsky, A. Neronov, O. Ruchayskiy, M. Shaposhnikov, Masses of active neutrinos in the $\nu M S M$ from x-ray astronomy, JETP Lett. (2006) 133 [arXiv:hep-ph/0601098].

[26] S. Dodelson, M. L. Widrow, Sterile neutrinos as dark matter, Phys. rev. Lett. 72 (1994) 17 [arXiv:hep-ph/9303287].

[27] S. Tremaine, J. E. Gunn, Dynamical role of light neutral leptons in cosmology, Phys. Rev. Lett. 42 (1979) 407.

[28] K. Abazajian, Linear cosmological structure limits on warm dark matter, Phys. Rev. D 73 (2006) 063513 [arXiv:astro-ph/0512631].

[29] M. Viel, J. Lesgourgues, M. G. Haehnelt, S. Matarrese, A. Riotto, Can Sterile Neutrinos Be Ruled Out as Warm Dark Matter Candidates?, Phys. Rev. Lett. 97 (2006) 063513 [arXiv:astro-ph/0605706].

[30] U. Seljak, A. Makarov, P. McDonald, H. Trac, Can Sterile Neutrinos Be the Dark Matter?, Phys. Rev. Lett. 97 (2006) 191303 [arXiv:astro-ph/0602430]. 
[31] E. Polisensky, M. Ricotti, Constraints on the dark matter particle mass from the number of Milky Way satellites, Phys. Rev. D 83 (2011) 043506 [arXiv:1004.1459].

[32] S. Horiuchi, P. J. Humphrey, J. Oñorbe, K. Abazajian, M. Kaplinghat, S. Garrison-Kimmel, Sterile neutrino dark matter bounds from galaxies of the Local Group, Phys. Rev. D 89 (2014) 025017 [arXiv:1311.0282].

[33] C. R. Watson, J. F. Beacom, H. Y:uksel, T. P. Walker, Direct $x$-ray constraints on sterile neutrino warm dark matter, Phys. Rev. D 74 (2006) 033009 [arXiv:astro-ph/0605424].

[34] A. Boyarsky, D. Iakubovskyi, O. Ruchayskiy, V. Savchenko, Constraints on decaying dark matter from XMM-Newton observations of M31, Mon. Not. Roy. Astron. Soc. 387 (2008) 1361 [arXiv:0709.2301].

[35] C. R. Watson, Z. Li, N. K. Polley, Constraining sterile neutrino warm dark matter with Chandra observations of the Andromeda galaxy, JCAP 1203, (2012) 018 [arXiv:1111.4217].

[36] S. P. Mikheyev, A. Yu.Smirnov, Resonance enhancement of oscillations in matter and solar neutrino spectroscopy, Yad. Fiz. 42 (1985) 1441.

[37] L. Wolfenstein, Neutrino oscillations in matter, Phys. Rev. D 17 (1978) 2369.

[38] X. Shi, G. M. Fuller, New Dark Matter Candidate: Nonthermal Sterile Neutrinos, Phys. Rev. Lett. 83 (1999) 3120 [arXiv:astro-ph/9810076].

[39] M. Laine, M. Shaposhnikov, Sterile neutrino dark matter as a consequence of MSM-induced lepton asymmetry, JCAP 0806 (2008) 031 [arXiv:0804.4543].

[40] K. M. Smith, W. Hu, M. Kaplighat, Cosmological information from lensed CMB power spectra, Phys. Rev. D 74 (2006) 123002 [astro-ph/0607315].

[41] Y-Z. Chu, M. Cirelli, Sterile neutrinos, lepton asymmetries, primordial elements: how much of each?, Phys. Rev. D 74 (2006) 085015 [astro-ph/0608206].

[42] D. Kirilova, Lepton Asymmetry and Neutrino Oscillations Interplay, Hyperfine Interactions 215 (2013) 111 [arXiv:1302.2923].

[43] A. Boyarsky, J. Lesgourgues, O. Ruchayskiy, M. Viel, Realistic Sterile Neutrino Dark Matter with KeV Mass does not Contradict Cosmological Bounds Phys. Rev. Lett. 102 (2009) 201304 [arXiv:0812.3256].

[44] K. Abazajian, G. M. Fuller, W. H. Tucker, Direct Detection of Warm Dark Matter in the X-Ray, Astrophys. J. 562 (2001) 593 [arXiv:astro-ph/0106002].

[45] A. Kusenko, Sterile Neutrinos, Dark Matter, and Pulsar Velocities in Models with a Higgs Singlet, Phys. Rev. Lett. 97 (2006) 241301 [arXiv:hep-ph/0609081].

[46] M. Shaposhnikov, I. Tkachev, The $\nu M S M$, inflation, and dark matter, Phys. Lett. B 639 (2006) 414 [arXiv:hep-ph/0604236].

[47] F. L. Bezrukov, D. S. Gorbunov, Relic gravity waves and $7 \mathrm{keV}$ dark matter from a GeV scale inflaton, Phys. Lett. B 736 (2014) 494 [arXiv:1403.4638].

[48] A. Merle, V. Niro, D. Schmidt, New production mechanism for keV sterile neutrino Dark Matter by decays of frozen-in scalars, JCAP 03 (2014) 028 [arXiv:1306.3996].

[49] A. Merle, A. Schneider, Production of Sterile Neutrino Dark Matter and the 3.5 keV line (2014), arXiv:1409.6311.

[50] Bulbul, E., Markevitch, M., Foster, A., Smith, R. K., Loewenstein,M., et al., Detection of an Unidentified Emission Line in the Stacked X-ray Spectrum of Galaxy Clusters, Astrophys.J., 789 (2014) 13 [arXiv:1402.2301].

[51] Boyarsky, A., Ruchayskiy, O., Iakubovskyi, D., \& Franse, J., An unidentified line in X-ray spectra of the Andromeda galaxy and Perseus galaxy cluster, Phys. Rev. Lett. 113 (2014) 25 
251301 [arXiv:1402.4119].

[52] Riemer-Sorensen, S. Questioning a $3.5 \mathrm{keV}$ dark matter emission line, arXiv:1405.7943

[53] Boyarsky, A., Franse, J., Iakubovskyi, D., \& Ruchayskiy, O. Checking the dark matter origin of $3.53 \mathrm{keV}$ line with the MilkyWay center, arXiv: 1408.2503

[54] Jeltema, T. E., \& Profumo, S. Dark matter searches going bananas: the contribution of Potassium (and Chlorine) to the $3.5 \mathrm{keV}$ line, [arXiv: 1408.1699].

[55] M. Loewenstein, A. Kusenko, P. L. Biermann, New Limits on Sterile Neutrinos from Suzaku Observations of the Ursa Minor Dwarf Spheroidal Galaxy, Astrphys.J. 700 (2009) 426 [arXiv:0812.2710].

[56] Urban, O., Werner, N., Allen, S. W., Simionescu, A., Kaastra, J. S., Strigari, L. E., A Suzaku Search for Dark Matter Emission Lines in the X-ray Brightest Galaxy Clusters, arXiv: 1411.0050 .

[57] Malyshev, D., Neronov, A., \& Eckert, D. Constraints on $3.55 \mathrm{keV}$ line emission from stacked observations of dwarf spheroidal galaxies, Physical Review D 90 (2014) Issue 10 id.1035062014 [arXiv:1408.3531].

[58] Anderson, M. E., Churazov, E., \& Bregman, J. N., Non-Detection of X-Ray Emission From Sterile Neutrinos in Stacked Galaxy Spectra, arXiv: 1408.4115.

[59] Boyarsky, A., Franse, J., Iakubovskyi, D., \& Ruchayskiy, O., Comment on the paper "Dark matter searches going bananas: the contribution of Potassium (and Chlorine) to the $3.5 \mathrm{keV}$ line" by T. Jeltema and S. Profumo, arXiv: 1408.4388.

[60] Bulbul, E., Markevitch, M., Foster, A., Smith, R. K., Loewenstein,M., Comment on "Dark matter searches going bananas: the contribution of Potassium (and Chlorine) to the $3.5 \mathrm{keV}$ line", arXiV:1409.4143.

[61] Carlson, E., Jeltema, T., Profumo, S., Where do the $3.5 \mathrm{keV}$ photons come from? A morphological study of the Galactic Center and of Perseus [arXiv:1411.1758].

[62] K. N. Abazajian, Resonantly Produced 7 keV Sterile Neutrino Dark Matter Models and the Properties of Milky Way Satellites, Phys. Rev. Lett. 112 (2014) 161303 [arXiv:1403.0954].

[63] L. A. Popa, A. Vasile, Constraints on non-thermal dark matter from PLANCK lensing extraction, JCAP 10 (2007) 017 [arXiv:0708.2030].

[64] Planck Collaboration; P. A. R. Ade et al., Planck 2013 results. XVII. Gravitational lensing by large-scale structure, Astron. 83 Astrophys. 571 (2014) 39 [arXiv:1303.5077].

[65] A. Caramete, L. A. Popa, Cosmological evidence for leptonic asymmetry after Planck, JCAP 02 (2014) 012 [arXiv:1311.3856].

[66] K. Abazajian, N. F. Bell, G. M. Fuller, Y. Y. Y. Wong, Cosmological lepton asymmetry, primordial nucleosynthesis and sterile neutrinos, Phys. Rev. D 72 (2005) 063004 [arXiv:astro-ph/0410175].

[67] E. Grohs, G. M. Fuller, C. T. Kishimoto, M. W. Paris, Effects of neutrino rest mass on $N_{e} x t e f f$ and ionization equilibrium freeze-out, arXiv:1412.6875.

[68] E. Grohs, G. M. Fuller, C. T. Kishimoto, M. W. Paris, Probing neutrino physics with a self-consistent treatment of the weak decoupling, nucleosynthesis, and photon decoupling epochs, JCAP 05, (2015) 017 [arXiv:1502.02718].

[69] C. T. Kishimoto, G. M. Fuller, G. J. Smith, Coherent Active-Sterile Neutrino Flavor Transformation in the Early Universe, Phys. Rev. Lett. 97 (2006) 141301 [arXiv:astro-ph/0607403].

[70] C. J. Smith, G. M. Fuller, C. T. Kishimoto, K. N. Abazajian, Light element signatures of 
sterile neutrinos and cosmological lepton numbers, Phys. Rev. D 74 (2006) 085008 [arXiv:astro-ph/0608377]

[71] A. D. Dolgov, Neutrinos in cosmology, Phys. Rep. 370 (2002) 333 [hep-ph/0202122].

[72] P. D. Serpico and G. G. Raffelt, Lepton asymmetry and primordial nucleosynthesis in the era of precision cosmology, Phys. Rev. D 71 (2005) 127301 [astro-ph/0506162].

[73] V. Simha and G. Steigman, Constraining the universal lepton asymmetry, JCAP 08 (2008) 011 [arXiv:0806.0179].

[74] Y. Y. Wong, Analytical treatment of neutrino asymmetry equilibration from avor oscillations in the early universe, Phys. Rev. D 66 (2002) 025015 [hep-ph/0203180].

[75] K. N. Abazajian, J. F. Beacom and N. F. Bell, Stringent constraints on cosmological neutrino-antineutrino asymmetries from synchronized avor transformation, Phys. Rev. D 66 (2002) 013008 [astro-ph/0203442].

[76] G. Mangano, G. Miele, S. Pastor, O. Pisanti, S. Sarikas, Updated BBN bounds on the cosmological lepton asymmetry for non-zero $\theta$, Phys. Lett B 708 (2012) 1 [arXiv:1110.4335].

[77] E. Castorina, F. Urbano; M. Lattanzi, J. Lesgourgues, G. Mangano, A. Melchiorri, S. Pastor, Cosmological lepton asymmetry with a nonzero mixing angle $\theta_{13}$, Phys. Rev. D 86 (2012) 023517 [arXiv:1204.2510].

[78] G. Mangano, G. Miele, S. Pastor, O. Pisanti, S. Sarikas, Constraining the cosmic radiation density due to lepton number with Big Bang Nucleosynthesis, JCAP 03 (2011) 035 [arXiv:1011.0916].

[79] D. Boyanovsky, C. Ho, Sterile neutrino production via active-sterile oscillations: the quantum Zeno effect, JHEP 0707 (2007) 030 [arXiv:hep-ph/0612092].

[80] S. Seager, D. D. Sasselov, D. Scott, A New Calculation of the Recombination Epoch ApJ 523 (1999) L1 [astro-ph/9909275].

[81] A. Lewis, A. Challinor and A. Lasenby, Efficient Computation of CMB anisotropies in closed FRW models, Astrophys. J. 538 (2000) 473 [astro-ph/9911177]. ${ }^{1}$

[82] J. Lesgourgues, S. Pastor, Cosmological implications of a relic neutrino asymmetry, Phys. Rev. D 60 (1999) 103521 [hep-ph/9904411].

[83] K. Ichiki, M. Yamaguchi and J. Yokoyama,Lepton asymmetry in the primordial gravitational wave spectrum, Phys. Rev D 75 (2007) 084017 [hep-ph/0611121].

[84] L. A. Popa and A. Vasile, WMAP ve-year constraints on lepton asymmetry and radiation energy density: implications for Planck, JCAP 06 (2008) 028 [arXiv:0804.2971].

[85] W. Hu, D. Scott, N. Sugiyama and M. White, The Effect of Physical Assumptions on the Calculation of Microwave Background Anisotropies, Phys. Rev. D 52 (1995) 5498 [astro-ph/9505043].

[86] R. Trotta and A. Melchiorri, Indication for Primordial Anisotropies in the Neutrino Background from WMAP and SDSS, Phys. Rev Lett. 95 (2005) 011305 [astro-ph/0412066].

[87] O. Pisanti, A. Cirillo, S. Esposito, F. Iocco, G. Mangano, G. Miele and P.D. Serpico, PArthENoPE: Public algorithm evaluating the nucleosynthesis of primordial elements, Computer Physics Communications 178 (2008) 956 [arXiv: 0705.0290].

[88] J. P. Kneller and G. Steigman, BBN for pedestrians, New Journal of Physics 6 (2004) 117 [astro-ph/0406320].

[89] A. Lewis and S. Bridle, Cosmological parameters from CMB and other data: A Monte Carlo

\footnotetext{
${ }^{1}$ http://camb.info/
} 
approach, Phys. Rev. D 66 (2002) 103511 [astro-ph/0205436]. ${ }^{2}$

[90] C. L. Bennet et al., Nine-year Wilkinson Microwave Anisotropy Probe (WMAP) Observations: Final Maps and Results, Astrophys. J. Suppl. 208 (2013) 20 [arXiv:1212.5225].

[91] Planck Collaboration, P. A. R. Ade et al., Planck 2013 results. XV. CMB power spectra and likelihood, Astron. \& Astrophys. 571, id.A15 60 [arXiv: 1303.5075].

[92] Planck Collaboration, P. A. R. Ade et al., Planck 2013 results. XVII. Gravitational lensing by large-scale structure, Astron. 86 Astrophys. 571 (2014) 39 [arXiv: 1303.5077].

[93] S. Das et al., The Atacama Cosmology Telescope: Temperature and Gravitational Lensing Power Spectrum Measurements from Three Seasons of Data, JCAP 04 (2014) 014 [arXiv: 1301.1037].

[94] J. Dunkley et al., The Atacama Cosmology Telescope: likelihood for small-scale CMB data, JCAP 07 (2013) 025 [arXiv: 1301.0776].

[95] R. Keisler et al., A Measurement of the Damping Tail of the Cosmic Microwave Background Power Spectrum with the South Pole Telescope, Astrophys. J. 743 (2011) 17 [arXiv: 1105.3182].

[96] C. L. Reichardt et al., A measurement of secondary cosmic microwave background anisotropies with two years of South Pole Telescope observations, Astropys J. 755 (2012) 70 [arXiv: 1111.0932].

[97] W. J. Percival et al., Baryon Acoustic Oscillations in the Sloan Digital Sky Survey Data Release 7 Galaxy Sample, Mon. Not. Roy. Astron. Soc., 401 (2010) 2148 [arXiv: 0907.1660].

[98] Sloan Digital Sky Survey Collaboration, K. Abazajian et al., The Seventh Data Release of the Sloan Digital Sky Survey, Astrophys. J. Suppl. 182 (2009) 543 [arXiv: 0812.0694].

[99] N. Padmanabhan, X. Xu, D. J. Eisenstein, R. Scalzo, A. J. Cuesta, K. T. Mehta and E. Kazin, A 2\% Distance to $z=0.35$ by Reconstructing Baryon Acoustic Oscillations - I : Methods and Application to the Sloan Digital Sky Survey, Mon. Not. Roy. Astron. Soc. 427 (2012) 2132 [arXiv: 1202.0090].

[100] SDSS-III Collaboration, C. P. Ahn et al., The Ninth Data Release of the Sloan Digital Sky Survey: First Spectroscopic Data from the SDSS-III Baryon Oscillation Spectroscopic Survey, Astrophys. J. Suppl. 203 (2012) 21 [arXiv: 1207.7137].

[101] L. Anderson et al., The clustering of galaxies in the SDSS-III Baryon Oscillation Spectroscopic Survey: baryon acoustic oscillations in the Data Release 9 spectroscopic galaxy sample, Mon. Not. Roy. Astron. Soc. 427 (2012) 3435 [arXiv: 1203.6594].

[102] D. H. Jones et al., The 6dF Galaxy Survey: final redshift release (DR3) and southern large-scale structures, Mon. Not. Roy. Astron. Soc. 399 (2009) 683 [arXiv: 0903.5451].

[103] F. Beutler, C. Blake, M. Colless, D. H. Jones, L. Staveley-Smith, L. Campbell, Q. Parker, W. Saunders, F. Watson, The 6dF Galaxy Survey: baryon acoustic oscillations and the local Hubble constant, Mon. Not. Roy. Astron. Soc. 416 (2011) 3017 [arXiv: 1106.3366].

[104] E. Aver, K. Olive, E.D. Skillman, An MCMC determination of the primordial helium abundance, JCAP (1204 (2012) 004 [arXiv: 1112.3713].

\footnotetext{
${ }^{2}$ http://cosmologist.info/cosmomc/
} 